\title{
The Aetiology of Delay to Commencement of Adjuvant Chemotherapy following Colorectal Resection
}

\author{
G. S. Simpson, ${ }^{1}$ R. Smith, ${ }^{1}$ P. Sutton, ${ }^{1,2}$ A. Shekouh, ${ }^{1}$ C. McFaul, ${ }^{1}$ \\ M. Johnson, ${ }^{1}$ and D. Vimalachandran ${ }^{1}$ \\ ${ }^{1}$ Countess of Chester Hospital NHS Foundation Trust, Countess of Chester Health Park, Liverpool Road, Chester CH2 1UL, UK \\ ${ }^{2}$ University of Liverpool, Liverpool, Merseyside L69 3BX, UK \\ Correspondence should be addressed to G. S. Simpson; gregorysimpson@doctors.org.uk
}

Received 21 October 2013; Accepted 12 February 2014; Published 17 March 2014

Academic Editor: S. Curley

Copyright (C) 2014 G. S. Simpson et al. This is an open access article distributed under the Creative Commons Attribution License, which permits unrestricted use, distribution, and reproduction in any medium, provided the original work is properly cited.

\begin{abstract}
Purpose. Timely administration of adjuvant chemotherapy following colorectal resection is associated with improved outcome. We aim to assess the factors which are associated with delay to adjuvant chemotherapy in patients who underwent colorectal resection as part of an enhanced recovery protocol. Method. A univariate and multivariate analysis of patient data collected as part of a prospectively maintained database of colorectal cancer patients between 2007 and 2012. Results. 166 patients underwent colorectal resection followed by adjuvant chemotherapy. Median postoperative hospital stay was 6 days, and time to commencement of adjuvant chemotherapy was 50 days. Longer inpatient stay correlated with increased time to adjuvant chemotherapy $(P=0.05)$. Factors found to be independently associated with duration of hospital stay and time to commencement of adjuvant chemotherapy included stoma formation $(P=0.032)$, anastaomotic leak $(P=0.027)$, and preoperative albumin $(P=0.027)$. The use of laparoscopic surgery was associated with shorter time to adjuvant chemotherapy but did not reach significance $(P=0.143)$. Conclusion. A number of independent variables associated with delay to adjuvant therapy previously not described have been identified. Further work may be required to elucidate the effect that these variables have on long-term outcome.
\end{abstract}

\section{Introduction}

Colon and rectal cancer is a common malignancy worldwide, having the third highest incidence of all cancers with around 1 million diagnoses worldwide each year [1]. Multimodality treatment strategies are employed in the management of colorectal malignancy; with neoadjuvant and adjuvant treatments complimenting the mainstay of treatment-surgical resection.

The use of adjuvant chemotherapy (AC) following surgical resection of colorectal cancer has been shown to improve outcome [2-5]. Adjuvant chemotherapy has been advocated in patients with stage II disease associated with adverse disease features including T4 disease, perforation or obstruction [6], and in all patients with stage III disease [7].

The timing of administration of adjuvant chemotherapy following surgical resection has been proposed as a factor that potentially affects overall outcome, although this has not been proven conclusively. Some studies have demonstrated that initiation of chemotherapy occurring more promptly following surgical resection is being associated with improved outcome [8-10]. A meta-analysis found poorer outcomes if chemotherapy is administered 8 weeks or more after surgery [11], whilst another meta-analysis has reported a decrease in overall survival of $14 \%$ for each 4 -week delay in administration of adjuvant chemotherapy [12].

Multiple factors dictating postoperative course and outcome in colorectal cancer have been identified including markers of the extent of systemic inflammatory response such as the Glasgow Prognostic Score (GPS), C-reactive protein, and albumin [13-15]. In addition, physiological parameters [16], patient comorbidity [17], and operative strategy [18, 19] have been shown to influence postoperative course and outcome. In contrast, limited information regarding the factors associated with increased delay to commencement of adjuvant therapy is available; however, age and race have been 
TABLE 1: Patient demographics and operative details.

\begin{tabular}{lc}
\hline Number of patients identified & $\mathbf{1 6 6}$ \\
Gender: men & $112(67 \%)$ \\
Age: median (IQR) & $66(61$ to 73$)$ years \\
Comorbidity & $91(55 \%)$ \\
BMI: median (IQR) & $27.3(24.2$ to 30.3$)$ \\
Neoadjuvant chemoradiotherapy & \\
None & $124(75 \%)$ \\
Long course & $36(22 \%)$ \\
Short course & $6(3 \%)$ \\
Operation details & \\
Anterior resection & $63(38 \%)$ \\
Right hemicolectomy & $50(30 \%)$ \\
Left/sigmoid colectomy & $30(18 \%)$ \\
Abdominoperineal resection & $13(8 \%)$ \\
Hartmann's procedure & $6(4 \%)$ \\
Subtotal colectomy & $3(2 \%)$ \\
Panproctocolectomy & $1(<1 \%)$ \\
Mode of surgery & \\
Open & $124(75 \%)$ \\
Laparoscopic & $42(19 \%)$ \\
Converted & $10(6 \%)$ \\
Elective $:$ emergency & $153(92 \%): 13(8 \%)$ \\
Stoma required & $72(43 \%)$ \\
Preoperative bloods: median $(\mathrm{IQR})$ & \\
Haemoglobin $(\mathrm{g} / \mathrm{dL})$ & $13.0(11.9$ to 14.3$)$ \\
Platelets $\left(\times 10^{6} / \mathrm{mL}\right)$ & $249(218$ to 333$)$ \\
Neutrophils $\left(\times 10^{6} / \mathrm{mL}\right)$ & $4.6(3.6$ to 5.7$)$ \\
Lymphocytes $\left(\times 10^{6} / \mathrm{mL}\right)$ & $1.5(1.0$ to 2.0$)$ \\
Albumin (mg/L) & $38(35$ to 41$)$ \\
C-reactive protein $(\mathrm{mg} / \mathrm{L})$ & $3(2$ to 11$)$ \\
\hline &
\end{tabular}

IQR: interquartile range.

linked to delay in administration of adjuvant chemotherapy [20], whilst the occurrence of surgical complications has been associated with complete omission of adjuvant chemotherapy rather than delay of commencement [21].

Our aim is to identify factors which are associated with increased delay in administration of adjuvant chemotherapy in a cohort of patients undergoing curative resection for colorectal cancer.

\section{Patients and Methods}

An analysis of a prospectively maintained database containing details of all patients undergoing colorectal cancer resections from 2007 to 2012 was performed. All those with stages II-III colorectal cancer who received adjuvant chemotherapy following surgical resection were identified and included in the study. Relevant data pertaining to patient characteristics, operative strategy, complications, histology, biochemical parameters, and adjuvant therapy were extracted and analysed.
TABLE 2: Histological tumour characteristics.

\begin{tabular}{lc}
\hline Histology & \\
Tumour size: median (IQR) & \\
Differentiation & \\
Well/moderate & $146(88 \%)$ \\
Poor & $13(8 \%)$ \\
Complete response & $7(4 \%)$ \\
Node status & \\
N0 & $57(34 \%)$ \\
N1 & $72(43 \%)$ \\
N2 & $37(22 \%)$ \\
Median nodal yield & $14(10$ to 20$)$ \\
Median number of involved nodes & $3(1$ to 5$)$ \\
Median lymph node ratio & $0.18(0.10$ to 0.33$)$ \\
T stage & \\
T0/T1 & $12(7 \%)$ \\
T2 & $14(8 \%)$ \\
T3 & $92(55 \%)$ \\
T4 & $48(29 \%)$ \\
Resection margin status & \\
R0 & $160(94.4 \%)$ \\
R1 & $6(3.6 \%)$ \\
Vascular invasion & \\
Positive & $46(28 \%)$ \\
Negative & $120(72 \%)$ \\
\hline
\end{tabular}

2.1. Outcome Measures. The time period (days) between surgical resection and commencement of adjuvant chemotherapy was calculated. Pre- and postoperative variables, histology, and biochemical parameters were analysed as to know their influence on the time to administration of adjuvant chemotherapy.

2.2. Statistical Analysis. All continuous data were analysed with median, interquartile range, and 95\% confidence intervals. Nonparametric tests were employed for comparative purposes (Mann-Whitney $U$ test). The interval between surgery and commencement of adjuvant chemotherapy was analysed as time to event data using Cox regression to analyse continuous and categorical variables for univariate and multivariate analysis. Software used included StatView V5 (SAS Institute, Cary, NC).

\section{Results}

166 patients who underwent intended curative resection for colorectal adenocarcinoma followed by adjuvant chemotherapy were identified. Table 1 outlines the patient demographics and operative details for this patient cohort. Table 2 outlines the histological characteristics of the resected cancers. Preoperative blood was typically recorded within 24 hours of surgery-median time interval $=1$ day $(\mathrm{IQR}=1$ to 6$)$. 


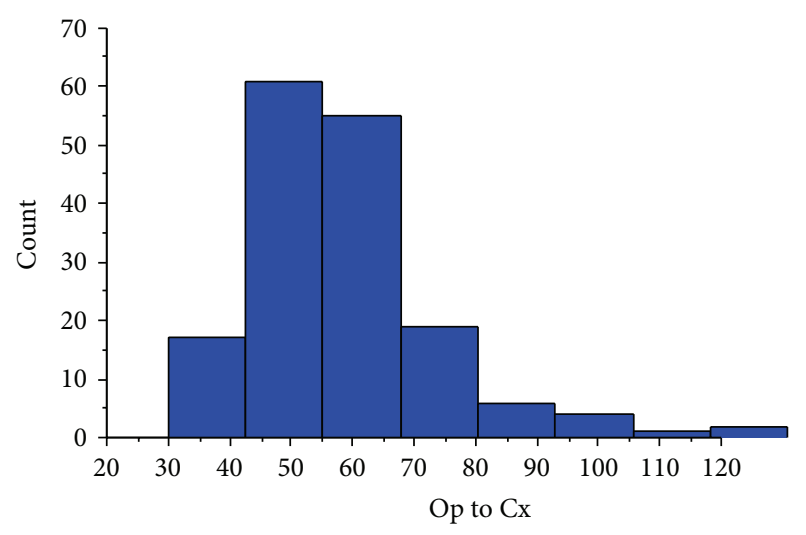

FIGURE 1: Distribution of time intervals from operation to commencement of chemotherapy (for inclusion in online publication only).

3.1. Postoperative Data. The median duration of hospital stay was $6(\mathrm{IQR}=5$ to 8$)$ days. Five patients $(3 \%)$ had a postoperative anastomotic leak; four of whom required further surgery. Two patients (1\%) had significant postoperative bleeding; one of whom required reoperation and one required readmission. The median time interval from hospital discharge to commencing chemotherapy was 50 $(\mathrm{IQR}=41$ to 58$)$ days. Patients with a longer postoperative inpatient stay exhibited a significant trend towards having a longer time interval from discharge to chemotherapy (linear regression; $t=1.94 ; P=0.050$ )

3.2. Interval from Operation to Chemotherapy. Overall, the median time interval from the date of surgery to date of commencing adjuvant chemotherapy was 58 days $(\mathrm{IQR}=$ 39 to 77). Figure 1 illustrates this distribution. No patients received chemotherapy within 30 days of surgery. 107 (64\%) patients received chemotherapy between 30 and 60 days of surgery and 59 (36\%) patients received chemotherapy after 60 days.

Table 3 demonstrates the relationship between the clinicopathological factors investigated and time from surgery to commencement of adjuvant chemotherapy (Cox regression). From this analysis preoperative hypoalbuminaemia, anastomotic leak, requirement for stoma, and increasing lymph node ratio were all identified as having a potential association with a longer wait to commencement of adjuvant chemotherapy $(P<0.100)$. Patients undergoing laparoscopic surgery exhibited a trend towards shorter time intervals to starting adjuvant chemotherapy but this failed to reach significance $(P=0.143)$. On multivariate Cox regression, all four factors were independently significant. Figure 2 illustrates the associations between these four variables and time to chemotherapy. Due to incomplete preoperative biochemical data in 7 cases, the final multivariate analysis included 159 patients.

3.3. Duration of Hospital Admission. All four variables identified from the above analysis were also found to demonstrate a significant association with increased duration of postoperative stay (Table 4). Alongside this, patients undergoing laparoscopic resections were found to have a shorter postoperative hospital stay than those undergoing open surgery (Mann-Whitney; $P<0.001$ ) —Figures 3 and 4 .

\section{Discussion and Conclusion}

Adjuvant chemotherapy is a key component in the treatment of colorectal cancer and is shown to improve survival [3-5]. Data assessing the effect of timing of adjuvant chemotherapy have shown an increased mortality in patients where administration of chemotherapy has been delayed beyond 60 days $[10,22]$. Only a small number of reports have demonstrated little effect of the timing of adjuvant chemotherapy following colorectal cancer resection on outcome [23, 24]. Recent meta-analyses have shown the benefit of early administration of chemotherapy, demonstrating a decrease in survival of $14 \%$ with every 4 -week increase in delay to chemotherapy following resection $[11,12]$. The finding of improved outcome with timely administration of adjuvant chemotherapy has also been documented in patients with cancer at other sites, most notably the breast [25-27] and pancreas [28].

Our data has identified multiple independently significant factors which are associated with increased delay to provision of adjuvant chemotherapy. Preoperative serum albumin has been shown to be inversely correlated with delay to commencement of adjuvant therapy. Similarly, our data has demonstrated that low preoperative serum albumin is associated with increased postoperative hospital stay. Albumin has previously been identified as a valuable preoperative marker linked to outcome following colorectal resection; however, no accounts are available in the literature showing it to be linked to timely receipt of adjuvant chemotherapy [1315]. Preoperative albumin represents a potential marker of disease severity which could represent the degree of disease progression relating to operative difficulty and extension of recovery time, detrimentally affecting timely administration of chemotherapy. Additionally, albumin acts as an indicator of poor preoperative nutritional repleteness and overall systemic upset, factors which will dictate postoperative recovery and readiness for adjuvant chemotherapy.

Anastomotic leak following colorectal resection has a profound impact upon postoperative course and has a known detrimental effect on recurrence and overall survival $[29,30]$, in addition to being associated with significant morbidity and often permanent stoma formation [31]. From our data, it can be seen that this impacts the duration of inpatient stay and its effect extends to timely administration of chemotherapy, with those patients experiencing an anastomotic leak further jeopardized by a delay in the commencement of their systemic therapy.

The use of a defunctioning stoma following colorectal resection has been associated with extended inpatient hospital stay and delay to chemotherapy in this patient population. Available literature shows the formation of a defunctioning stoma to carry morbidity in the early postoperative period $[32,33]$ and to extend postoperative hospital stay 


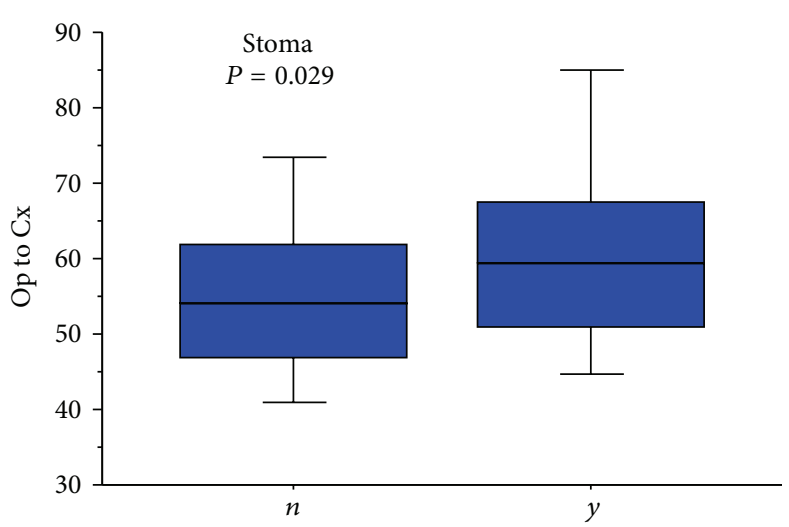

(a)

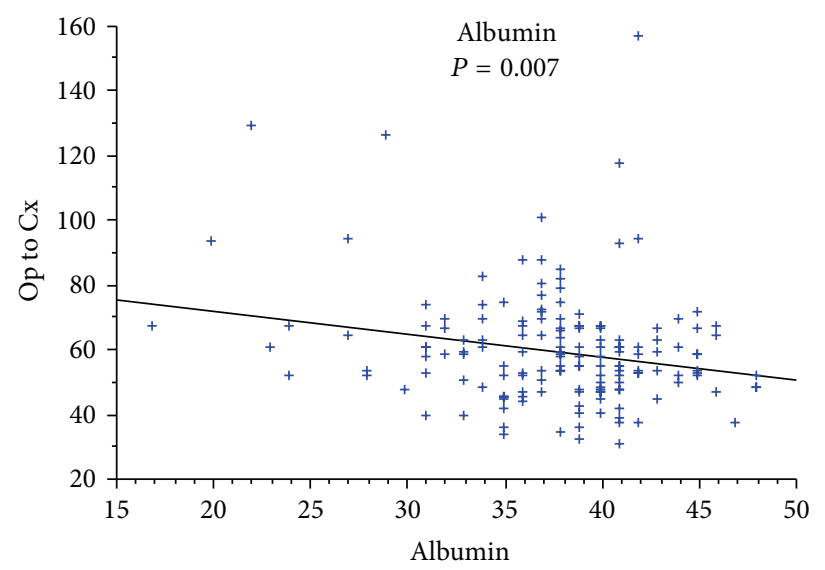

(c)

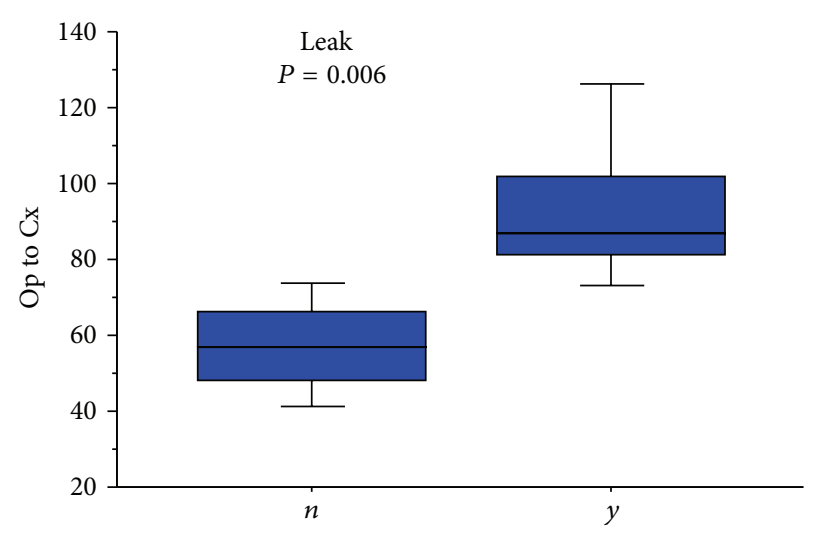

(b)

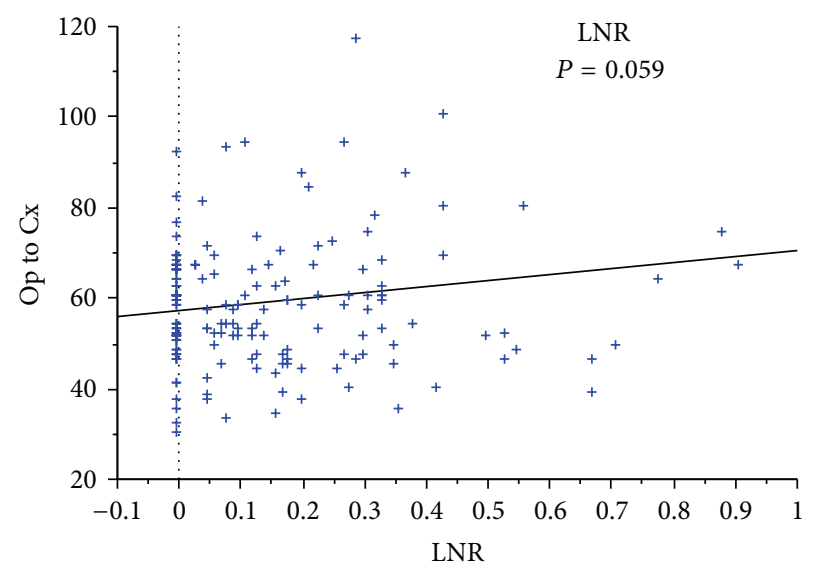

(d)

FIGURE 2: Relationship between requirement of stoma (a), anastomotic leak (b), preoperative serum albumin (c), and lymph node ratio (d) with time to adjuvant chemotherapy (categorical variables = Mann-Whitney; continuous variable = linear regression).

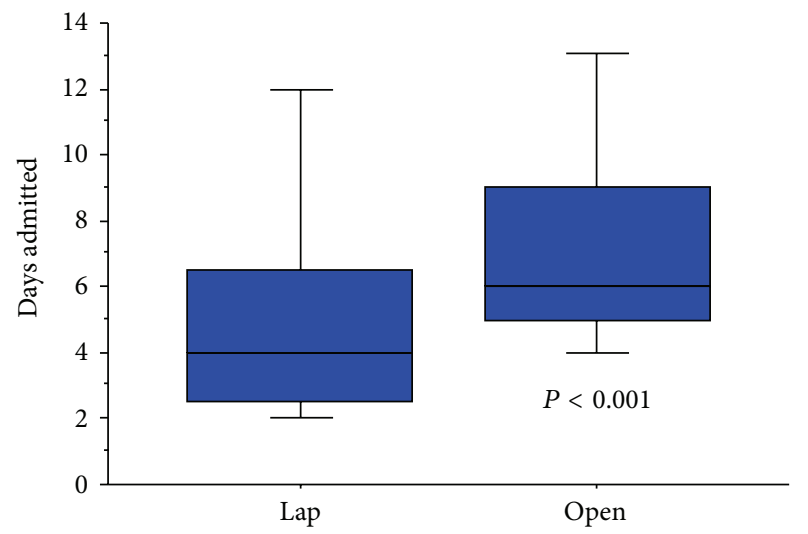

FIGURE 3: Association between laparoscopic surgery and shorter postoperative stay.

[34], mirroring our findings; however, the presence of a defunctioning stoma being associated with delay to adjuvant therapy has not been previously documented. The use of a defunctioning stoma is commonly associated with patients who have undergone preoperative chemoradiotherapy, rectal

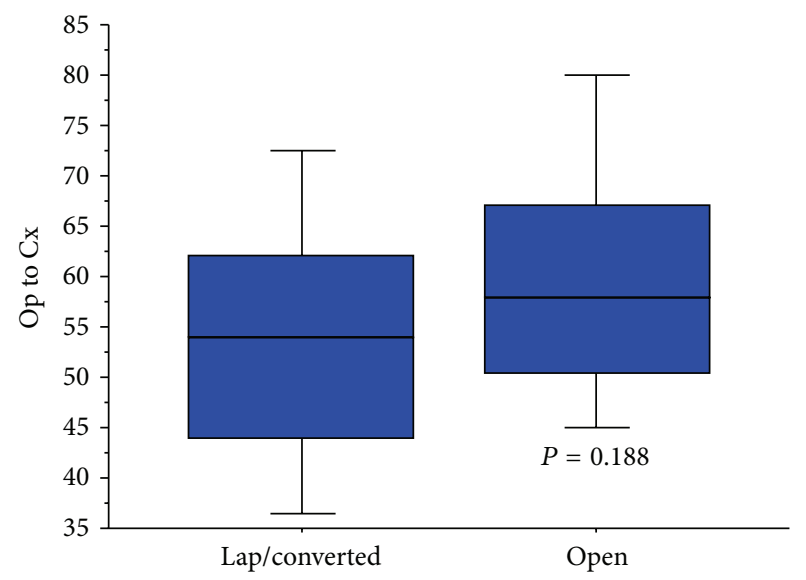

FIgURE 4: Association between approach to resection and time to adjuvant chemotherapy, laparoscopic/converted versus open (for inclusion in online publication only).

resections, and major or difficult resections, factors which may represent the cause of delay as opposed to the presence of a stoma. 
TABLE 3: Cox regression analysis of factors associated with time from surgery to commencement of adjuvant chemotherapy.

\begin{tabular}{|c|c|c|c|c|c|c|}
\hline & \multicolumn{3}{|c|}{ Univariate analysis } & \multicolumn{3}{|c|}{ Multivariate analysis $(n=159)$} \\
\hline & Hazard ratio $(95 \% \mathrm{CI})$ & $\chi^{2}$ & $P$ & Hazard ratio $(95 \% \mathrm{CI})$ & $\chi^{2}$ & $P$ \\
\hline Age & 0.993 (0.978 to 1.007$)$ & 0.973 & 0.324 & & & \\
\hline Gender (F) & $1.310(0.937$ to 1.832$)$ & 2.500 & 0.114 & & & \\
\hline BMI & 0.991 (0.961 to 1.023$)$ & 0.292 & 0.589 & & & \\
\hline Comorbidity (Y) & $1.083(0.789$ to 1.488$)$ & 0.247 & 0.619 & & & \\
\hline Neoadjuvant therapy (Y) & $0.889(0.625$ to 1.265$)$ & 0.425 & 0.514 & & & \\
\hline Laparoscopic procedure (Y) & 1.337 (0.906 to 1.973$)$ & 2.141 & 0.143 & & & \\
\hline Stoma $(Y)$ & 0.757 (0.555 to 1.031$)$ & 3.115 & 0.078 & 0.704 (0.512 to 0.970$)$ & 4.613 & 0.032 \\
\hline Tumour size & $1.002(0.994$ to 1.010$)$ & 0.180 & 0.672 & & & \\
\hline Differentiation (poor versus well/moderate) & 1.201 (0.679 to 2.122$)$ & 0.396 & 0.529 & & & \\
\hline T stage & $1.009(0.863$ to 1.179$)$ & 0.013 & 0.911 & & & \\
\hline $\mathrm{N}$ stage & $0.940(0.680$ to 1.299$)$ & 0.141 & 0.707 & & & \\
\hline Lymph node ratio & $0.494(0.215$ to 1.131$)$ & 2.785 & 0.095 & $0.408(0.168$ to 0.992$)$ & 3.915 & 0.048 \\
\hline Resection margin status $(+)$ & 0.917 (0.546 to 1.541$)$ & 0.107 & 0.744 & & & \\
\hline Vascular invasion $(+)$ & 1.151 (0.816 to 1.623$)$ & 0.645 & 0.422 & & & \\
\hline Anastomotic leak (Y) & $0.304(0.123$ to 0.749$)$ & 6.701 & 0.010 & $0.352(0.140$ to 0.887$)$ & 4.907 & 0.027 \\
\hline Preoperative haemoglobin & $1.006(0.915$ to 1.105$)$ & 0.013 & 0.908 & & & \\
\hline Preoperative platelets & $1.000(0.999$ to 1.002$)$ & 0.613 & 0.434 & & & \\
\hline Preoperative neutrophils & 0.977 (0.915 to 1.042$)$ & 0.518 & 0.472 & & & \\
\hline Preoperative lymphocytes & $0.966(0.824$ to 1.131$)$ & 0.187 & 0.666 & & & \\
\hline Preoperative C-reactive protein & $0.998(0.994$ to 1.001$)$ & 1.751 & 0.186 & & & \\
\hline Preoperative albumin & $1.036(1.007$ to 1.067$)$ & 5.933 & 0.015 & 1.034 (1.004 to 1.065$)$ & 4.916 & 0.027 \\
\hline Tumour site (colon versus rectum) & $1.092(0.802$ to 1.487$)$ & 0.311 & 0.577 & & & \\
\hline Emergency $(\mathrm{Y})$ & $0.755(0.426$ to 1.339$)$ & 0.925 & 0.336 & & & \\
\hline
\end{tabular}

TABLE 4: Multivariate Cox regression analysis of hypoalbuminaemia, anastomotic leak, requirement for stoma, lymph node ratio, and association with duration of postoperative admission (for inclusion in online publication only).

\begin{tabular}{lccc}
\hline & Hazard ratio $(95 \%$ CI $)$ & $\chi^{2}$ & $P$ \\
\hline Albumin & $1.044(1.012$ to 1.076$)$ & 7.573 & $\mathbf{0 . 0 0 6}$ \\
Anastomotic leak $(\mathrm{Y})$ & $0.127(0.042$ to 0.388$)$ & 13.150 & $<.0013$ \\
Stoma $(\mathrm{Y})$ & $0.609(0.437$ to 0.848$)$ & 8.613 & $\mathbf{0 . 0 0 3}$ \\
Lymph node ratio & $0.264(0.102$ to 0.686$)$ & 7.479 & $\mathbf{0 . 0 0 6}$ \\
\hline
\end{tabular}

Lymph node ratio defined as the number of involved lymph nodes divided by the total nodal yield has been proposed as a valuable prognostic indicator in colorectal cancer with studies showing that poorer long-term outcome is associated with an increasing lymph node ratio $[35,36]$ potentially as a result of more aggressive tumour biology in those tumours with higher lymph node ratios. Lymph node ratio in this study may represent those patients who have required a more extensive surgical dissection or are more systemically unwell as a consequence of their more aggressive malignancy and thus are more likely to experience a greater time to commencement of adjuvant chemotherapy.

Whilst the effect of delayed chemotherapy has been investigated, research into the aetiology of such a delay has been minimal. In the available evidence, factors which have previously been cited as having an association with increased delay to commencement of chemotherapy include advanced age, patient comorbidity, tumour grade, marital status, postoperative stay, and race [20, 22]. Our findings are not consistent with these previously documented associations. Administration of neoadjuvant therapies, tumour characteristics, patient comorbidity, age, and sex do not have a significant effect on the timely provision of adjuvant therapy in our data.

This study has identified a number of independently significant variables which are associated with delay to administration of adjuvant chemotherapy. The variables identified have not previously been described in the literature. Interestingly, the use of a laparoscopic approach to colorectal resection has been seen to yield a shorter wait to commencement of chemotherapy, although this did not achieve significance. This represents a potential further advantage of laparoscopic surgery in addition to shorter inpatient stay, postoperative pain, cost-effectiveness, and recovery time previously described [37-39]. This study is potentially limited by the retrospective nature of the data analysis. 
The importance of timely administration of adjuvant chemotherapy following surgical resection has been identified as of importance in a number of specialties and its benefit has been made evident in colorectal cancer. Vigilance regarding prompt administration of adjuvant chemotherapy to colorectal cancer patients following surgical resection should be promoted, with colorectal teams providing this aspect of treatment as promptly as possible following surgical resection. Our study demonstrates a number of factors associated with delay in receiving adjuvant chemotherapy and may be used to identify patients who are at risk of delayed adjuvant chemotherapy so that this may be addressed in preoperative and intraoperative treatment decisions.

\section{What Does This Paper Add to the Literature?}

We present a number of previously undescribed variables associated with delay to adjuvant chemotherapy. We show that extended postoperative stay is related to delay to commencement of adjuvant chemotherapy.

\section{Conflict of Interests}

The authors declare that there is no conflict of interests regarding the publication of this paper.

\section{Authors' Contribution}

Gregory S. Simpson contributed in conception and design, data collection and interpretation, and drafting of paper. Richard Smith contributed in design, data collection and interpretation, and drafting of paper. Paul Sutton contributed in data interpretation and drafting of paper. Ali R. Shekouh contributed in drafting of paper. Christopher D. McFaul contributed in drafting of paper; final approval of paper is to be submitted. Michael A. Johnson contributed in drafting of paper; final approval of paper is to be submitted. Dale Vimalachandran (senior author) contributed in design and drafting of paper; final approval of paper is to be submitted.

\section{References}

[1] D. M. Parkin, F. Bray, J. Ferlay, and P. Pisani, "Global cancer statistics, 2002," Ca-A Cancer Journal for Clinicians, vol. 55, no. 2, pp. 74-108, 2005.

[2] QUASAR Collaborative Group, "Adjuvant chemotherapy versus observation in patients with colorectal cancer: a randomised study," The Lancet, vol. 370, no. 9604, pp. 2020-2029, 2007.

[3] J. Sakamoto, "Efficacy of oral adjuvant therapy after resection of colorectal cancer: 5-Year results from three randomized trials," Journal of Clinical Oncology, vol. 22, no. 3, pp. 484-492, 2004.

[4] S. Dubé, F. Heyen, and M. Jenicek, "Adjuvant chemotherapy in colorectal carcinoma: results of a meta- analysis," Diseases of the Colon and Rectum, vol. 40, no. 1, pp. 35-41, 1997.

[5] S. Gill, C. L. Loprinzi, D. J. Sargent et al., "Pooled analysis of fluorouracil-based adjuvant therapy for stage II and III colon cancer: who benefits and by how much?" Journal of Clinical Oncology, vol. 22, no. 10, pp. 1797-1806, 2004.
[6] A. Figueredo, M. E. Coombes, and S. Mukherjee, "Adjuvant therapy for completely resected stage II colon cancer," Cochrane Database of Systematic Reviews, vol. 16, no. 3, Article ID CD005390, 2008.

[7] A. B. Benson III, D. Schrag, M. R. Somerfield et al., "American society of clnical oncology recommendations on adjuvant chemotherapy for stage II colon cancer," Journal of Clinical Oncology, vol. 22, no. 16, pp. 3408-3419, 2004.

[8] D. Hershman, M. J. Hall, X. Wang et al., "Timing of adjuvant chemotherapy initiation after surgery for stage III colon cancer," Cancer, vol. 107, no. 11, pp. 2581-2588, 2006.

[9] I. S. Lima, Y. Yasui, A. Scarfe, and M. Winget, "Association between receipt and timing of adjuvant chemotherapy and survival for patients with stage III colon cancer in Alberta, Canada," Cancer, vol. 117, no. 16, pp. 3833-3840, 2011.

[10] U. D. Bayraktar, E. Chen, S. Bayraktar et al., "Does delay of adjuvant chemotherapy impact survival in patients with resected stage ii and iii colon adenocarcinoma?" Cancer, vol. 117, no. 11, pp. 2364-2370, 2011.

[11] G. Des Guetz, P. Nicolas, G. Perret, J. Morere, and B. Uzzan, "Does delaying adjuvant chemotherapy after curative surgery for colorectal cancer impair survival? A meta-analysis," European Journal of Cancer, vol. 46, no. 6, pp. 1049-1055, 2010.

[12] J. J. Biagi, M. J. Raphael, W. J. Mackillop, W. Kong, W. D. King, and C. M. Booth, "Association between time to initiation of adjuvant chemotherapy and survival in colorectal cancer a systematic review and meta-analysis," Journal of the American Medical Association, vol. 305, no. 22, pp. 2335-2342, 2011.

[13] M. J. Proctor, D. S. Morrison, D. Talwar et al., "A comparison of inflammation-based prognostic scores in patients with cancer. A Glasgow Inflammation Outcome Study," European Journal of Cancer, vol. 47, no. 17, pp. 2633-2641, 2011.

[14] R. Carruthers, L. M. Tho, J. Brown, S. Kakumanu, E. McCartney, and A. C. McDonald, "Systemic inflammatory response is a predictor of outcome in patients undergoing preoperative chemoradiation for locally advanced rectal cancer," Colorectal Disease, vol. 14, no. 10, pp. e701-e707, 2012.

[15] L. H. Moyes, E. F. Leitch, R. F. McKee, J. H. Anderson, P. G. Horgan, and D. C. McMillan, "Preoperative systemic inflammation predicts postoperative infectious complications in patients undergoing curative resection for colorectal cancer," British Journal of Cancer, vol. 100, no. 8, pp. 1236-1239, 2009.

[16] C. H. Richards, E. F. Leitch, P. G. Horgan, J. H. Anderson, R. F. McKee, and D. C. McMillan, "The relationship between patient physiology, the systemic inflammatory response and survival in patients undergoing curative resection of colorectal cancer," British Journal of Cancer, vol. 103, no. 9, pp. 1356-1361, 2010.

[17] P. O. Hendry, J. Hausel, J. Nygren et al., "Determinants of outcome after colorectal resection within an enhanced recovery programme," British Journal of Surgery, vol. 96, no. 2, pp. 197205, 2009.

[18] S. Trastulli, R. Cirocchi, C. Listorti et al., "Laparoscopic vs open resection for rectal cancer: a meta-analysis of randomized clinical trials," Colorectal Disease, vol. 14, no. 6, pp. e277-e296, 2012.

[19] H. Ohtani, Y. Tamamori, T. Azuma et al., "A meta-analysis of the short- and long-term results of randomized controlled trials that compared laparoscopy-assisted and conventional open surgery for rectal cancer," Journal of Gastrointestinal Surgery, vol. 15, no. 8, pp. 1375-1385, 2011.

[20] W. Y. Cheung, B. A. Neville, and C. C. Earle, "Etiology of delays in the initiation of adjuvant chemotherapy and their impact on 
outcomes for stage II and III rectal cancer," Diseases of the Colon and Rectum, vol. 52, no. 6, pp. 1054-1063, 2009.

[21] S. Hendren, J. D. Birkmeyer, H. Yin, M. Banerjee, C. Sonnenday, and A. M. Morris, "Surgical complications are associated with omission of chemotherapy for stage III colorectal cancer," Diseases of the Colon and Rectum, vol. 53, no. 12, pp. 1587-1593, 2010.

[22] D. Hershman, M. J. Hall, X. Wang et al., "Timing of adjuvant chemotherapy initiation after surgery for stage III colon cancer," Cancer, vol. 107, no. 11, pp. 2581-2588, 2006.

[23] R. Zeig-Owens, S. T. Gershman, R. Knowlton, and J. S. Jacobson, "Survival and time interval from surgery to start of chemotherapy among colon cancer patients," Journal of Registry Management, vol. 36, no. 2, pp. 30-62, 2009.

[24] S. Ahmed, I. Ahmad, T. Zhu et al., "Early discontinuation but not the timing of adjuvant therapy affects survival of patients with high-risk colorectal cancer: a population-based study," Diseases of the Colon and Rectum, vol. 53, no. 10, pp. 1432-1438, 2010.

[25] N. Alkis, A. G. Durnali, U. Y. Arslan et al., "Optimal timing of adjuvant treatment in patients with early breast cancer," Medical Oncology, vol. 28, no. 4, pp. 1255-1259, 2011.

[26] C. Lohrisch, C. Paltiel, K. Gelmon et al., "Impact on survival of time from definitive surgery to initiation of adjuvant chemotherapy for early-stage breast cancer," Journal of Clinical Oncology, vol. 24, no. 30, pp. 4888-4894, 2006.

[27] D. L. Hershman, X. Wang, R. McBride, J. S. Jacobson, V. R. Grann, and A. I. Neugut, "Delay of adjuvant chemotherapy initiation following breast cancer surgery among elderly women," Breast Cancer Research and Treatment, vol. 99, no. 3, pp. 313-321, 2006.

[28] Y. Murakami, K. Uemura, T. Sudo et al., "Early initiation of adjuvant chemotherapy improves survival of patients with pancreatic carcinoma after surgical resection," Cancer Chemotherapy and Pharmacology, vol. 71, no. 2, pp. 419-429, 2013.

[29] M. A. Boccola, P. G. Buettner, W. M. Rozen et al., "Risk factors and outcomes for anastomotic leakage in colorectal surgery: a single-institution analysis of 1576 patients," World Journal of Surgery, vol. 35, no. 1, pp. 186-195, 2011.

[30] W. L. Law, H. K. Choi, Y. M. Lee, J. W. C. Ho, and C. L. Seto, "Anastomotic leakage is associated with poor longterm outcome in patients after curative colorectal resection for malignancy," Journal of Gastrointestinal Surgery, vol. 11, no. 1, pp. 8-15, 2007.

[31] A. A. Khan, J. M. D. Wheeler, C. Cunningham, B. George, M. Kettlewell, and N. J. M. Mortensen, "The management and outcome of anastomotic leaks in colorectal surgery," Colorectal Disease, vol. 10, no. 6, pp. 587-592, 2008.

[32] O. Akesson, I. Syk, G. Lindmark, and P. Buchwald, "Morbidity related to defunctioning loop ileostomy in low anterior resection," International Journal of Colorectal Disease, vol. 27, no. 12, pp. 1619-1623, 2012.

[33] V. L. Tsikitis, D. W. Larson, V. P. Poola et al., "Postoperative morbidity with diversion after low anterior resection in the era of neoadjuvant therapy: a single institution experience," Journal of the American College of Surgeons, vol. 209, no. 1, pp. 114-118, 2009.

[34] M. T. Cartmell, O. M. Jones, B. J. Moran, and T. D. Cecil, “A defunctioning stoma significantly prolongs the length of stay in laparoscopic colorectal resection," Surgical Endoscopy and Other Interventional Techniques, vol. 22, no. 12, pp. 2643-2647, 2008.
[35] K. P. Wong, J. T. C. Poon, J. K. M. Fan, and W. L. Law, "Prognostic value of lymph node ratio in stage III colorectal cancer," Colorectal Disease, vol. 13, no. 10, pp. 1116-1122, 2011.

[36] R. Rosenberg, J. Engel, C. Bruns et al., "The prognostic value of lymph node ratio in a population-based collective of colorectal cancer patients," Annals of Surgery, vol. 251, no. 6, pp. 1070-1078, 2010.

[37] M. Z. Li, L. B. Xiao, W. H. Wu, S. B. Yang, and S. Z. Li, "Metaanalysis of laparoscopic versus open colorectal surgery within fast-track perioperative care," Diseases of the Colon \& Rectum, vol. 55, no. 7, pp. 821-827, 2012.

[38] C. C. Jensen, L. M. Prasad, and H. Abcarian, "Cost-effectiveness of laparoscopic vs open resection for colon and rectal cancer," Diseases of the Colon \& Rectum, vol. 55, no. 10, pp. 1017-1023, 2012.

[39] M. M. Reza, J. A. Blasco, E. Andradas, R. Cantero, and J. Mayol, "Systematic review of laparoscopic versus open surgery for colorectal cancer," British Journal of Surgery, vol. 93, no. 8, pp. 921-928, 2006. 


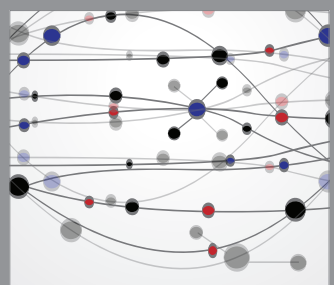

The Scientific World Journal
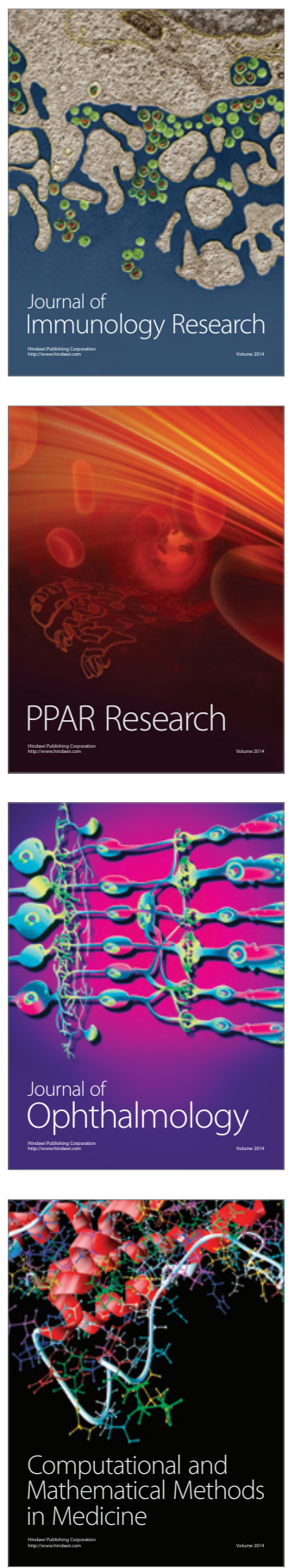

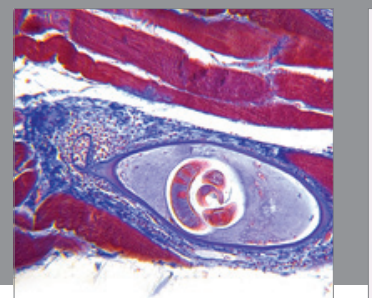

Gastroenterology

Research and Practice
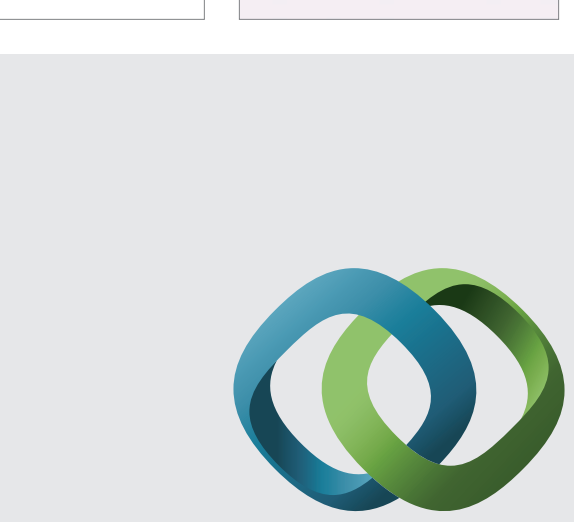

\section{Hindawi}

Submit your manuscripts at

http://www.hindawi.com
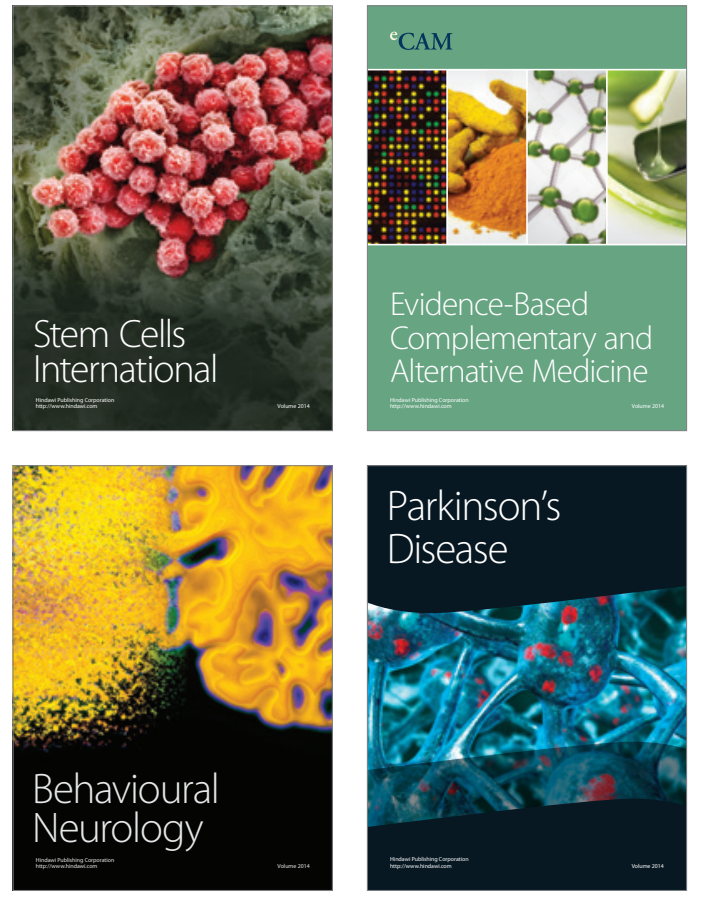
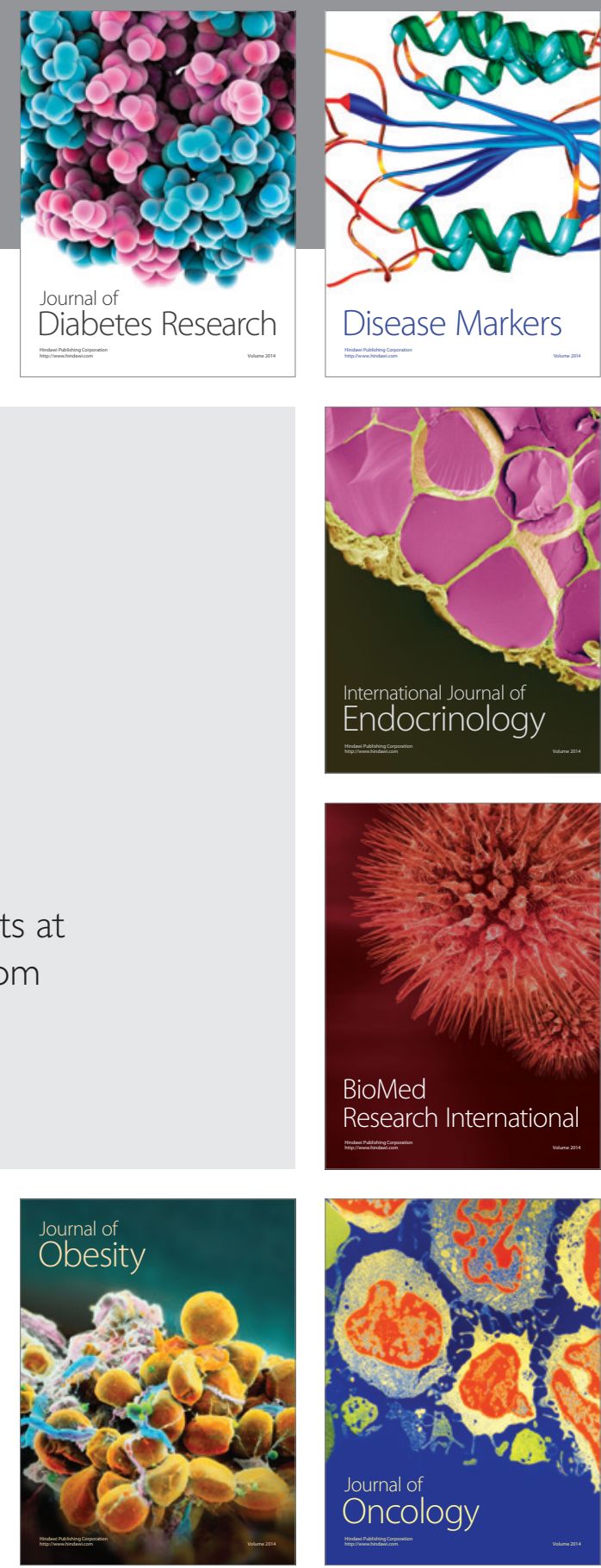

Disease Markers
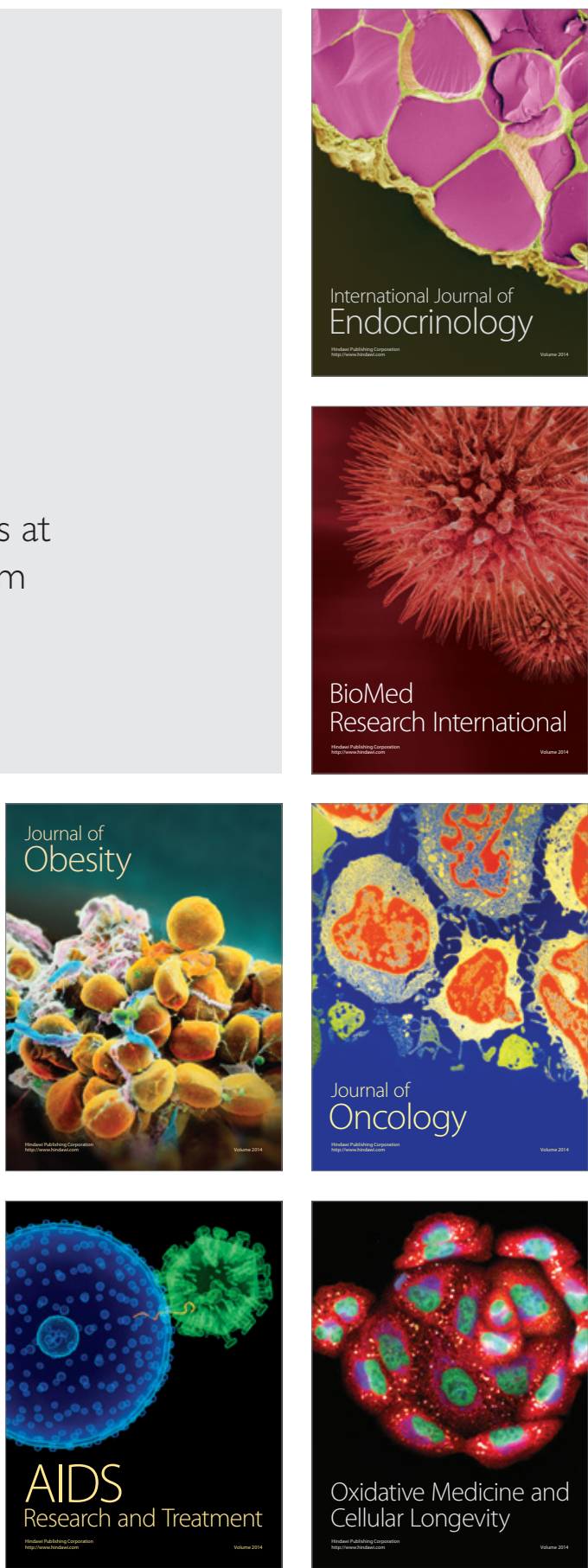